\title{
Analysis of ADAM17 Polymorphisms and Susceptibility to Sporadic Abdominal Aortic Aneurysm
}

\author{
You Lia Cheng Yang ${ }^{\text {b,e }}$ Guoda Ma ${ }^{a}$ Lili Cui ${ }^{a}$ Xuefeng Gu ${ }^{a}$ Yanyan Chen ${ }^{a}$ \\ Bin Zhao ${ }^{\mathrm{a}, \mathrm{c}}$ Haiyang Wang ${ }^{\mathrm{d}}$ Keshen $\mathrm{Li}^{\mathrm{a}}$ \\ aGuangdong Key Laboratory of Age-Related Cardiac and Cerebral Diseases, Affiliated Hospital of \\ Guangdong Medical College, Zhanjiang, 'D Department of Cardiac Surgery, Zhong Shan Hospital, Fu Dan \\ University, Shanghai Institute of Cardiovascular Disease, Shanghai, Institute of Neurology, Affiliated \\ Hospital of Guangdong Medical College, Zhanjiang, dDepartment of Vascular Surgery of General \\ Surgery, The First Affiliated Hospital of Harbin Medical University, Harbin, Heilongjiang, China; eThese \\ two authors contributed equally to this work
}

\section{Key Words}

ADAM17 • Abdominal aortic aneurysm • Polymorphism

\begin{abstract}
Background: Accumulating evidence suggests that the principal TNF- $\alpha$ converting enzyme, a disintegrin and metalloproteinase 17 (ADAM17), is involved in the development of human abdominal aortic aneurysm (AAA). However, the association between ADAM17 gene polymorphisms and AAA has not been explored. The present study was aimed to determine the association between ADAM17 promoter polymorphisms and AAA. Methods: A total of 316 patients with AAA and 306 age-matched healthy controls were enrolled in this study. Two ADAM17 promoter polymorphisms (rs12692386 and rs1524668) were determind. Realtime PCR was employed to detect the expression of ADAM17. Results: Overall, there was a significant difference in the frequency of the genotype rs12692386 between the AAA and control subjects $(P=0.0096)$. Furthermore, men with the rs12692386 AG genotype conferred a higher risk of developing AAA ( $P=0.0058)$. Additionally, the rs 12692386 mutated AG genotype of $A D A M 17$ was significantly associated with increased ADAM17 expression $(P=0.035)$ and TNF- $\alpha$ production $(P=0.042)$ in AAA patients. In contrast, the allele frequency of rs1524668 was not statistically associated with AAA. Conclusions: Our findings indicate a positive association between the rs12692386 polymorphism of ADAM17 and AAA. This new knowledge about ADAM17 identifies a role for ADAM17 in the pathophysiology of AAA and has important clinical implications with regard to potential therapeutics.
\end{abstract}

Guangdong Key Laboratory of Age-Related Cardiac and Cerebral Diseases, Affiliated Hospital of Guangdong Medical College, Zhanjiang 524001 (China) and Department of Vascular Surgery of General Surgery, The First Affiliated Hospital of Harbin Medical University, Harbin, Heilongjiang 150001 (China)

E-Mail likeshen1971@126.com and E-Mail haiyangwang811@163.com 


\section{Introduction}

Human abdominal aortic aneurysm (AAA) is the most common type of aneurysm in humans with a mortality rate associated with rupture as high as 90\% [1]. Multiple characteristics of AAA pathogenesis involve chronic inflammation [2], autoimmunity, increased neoangiogenesis [3], enhanced oxidative stress [4], and degradation of the extracellular matrix (ECM) [5]; however, the precise mechanisms of AAA formation and progression remain elusive [6].

Several studies have suggested that proinflammatory cytokines, especially tumor necrosis factor (TNF)- $\alpha$, play a large part in the etiology of AAA [7-9]. TNF- $\alpha$, a proinflammatory cytokine, is involved in the regulation of a wide spectrum of biological processes, including cell proliferation, differentiation, apoptosis, lipid metabolism, and coagulation. TNF- $\alpha$ levels were reported as increased in plasma and in the aorta in human AAA patients [9]. Blocking TNF- $\alpha$ attenuated aneurysm formation in $\mathrm{CaCl}_{2}$-induced AAA in mice through the inhibition of chronic inflammation and ECM disruption in the aortic wall; this result indicates that TNF- $\alpha$ plays a critical role in the pathogenesis of AAA [8]. TNF- $\alpha$ is generated via cleavage of the $32 \mathrm{kDa}$ transmembrane precursor by TNF- $\alpha$-converting enzyme (TACE), also referred to as a disintegrin and metalloproteinase (ADAM)-17. TACE belongs to the ADAMs family, which is involved in a number of biological processes, such as cell fate determination, cell migration, wound healing, neurite and axon guidance, heart development, immunity, cell proliferation, angiogenesis and inflammation [10]. TACE expression was increased in aortic lesions and a higher vascular shedding activity of TACE substrates (TNF and its receptors) was measured ex vivo from aortic explants of the apoE-/- mice $[9,11]$. These results highlight the predominant role of TNF shedding via TACE activity in the inflammatory reaction that occurs in the lesion [12]. A deficiency in TACE has been associated with reduced expression of pro-inflammatory/chemotactic cytokines and adhesion molecules, macrophage infiltration, VEGF-A expression and neoangiogenesis, MMP activation and ECM degradation [9]. In vivo evidence also demonstrated that a lack of TACE attenuated aneurysm formation in mice with AAA [13]. In summary, these lines of evidence have led us to formulate the hypothesis that ADAM17 could be of pathogenic significance in AAA.

The human ADAM17 gene is located on chromosome 2 and consists of 19 exons that span $55 \mathrm{~kb}$. ADAM17 polymorphisms have been investigated in relation to Alzheimer's disease [14], obesity [15] and Coronary artery disease [16]. Morange et al. [16] reported that the C-154A and Ser747Leu mutations of the ADAM17 gene are associated with TNF- $\alpha$ plasma levels and the risk of cardiovascular death, respectively. Junyent et al. [15] suggested that two ADAM17 polymorphisms, rs11684747 and rs10495563, are related to obesity and insulin resistance-related phenotypes (higher insulin and lower HDL-C concentrations). However, a genetic association between human ADAM17 polymorphisms and AAA has not yet been determined. Therefore, we carried out a case-control study to ascertain whether ADAM17 promoter polymorphisms are associated with AAA susceptibility.

\section{Materials and Methods}

\section{Study population}

We enrolled 316 cases that were randomly selected from AAA patients who were admitted to the Department of Vascular Surgery at the First Affiliated Hospital of Harbin Medical University from 2004 to 2012. Their ages ranged from 45 to 86 years, and 242 cases were male, while 74 cases were female. The AAA diameter of all patients ranged from 32 to $78 \mathrm{~mm}$. AAA was diagnosed by using computed tomography of the abdomen, which can detect dilatation of the abdominal aorta. Patients with inflammatory disorders, connective tissue disease, chronic renal failure, malignant disease or familial AAAs were excluded from the study. Sixty-nine patients with a maximal aortic diameter greater than $50 \mathrm{~mm}$ were admitted for elective surgery. Three hundred and six sex- and age-matched control subjects from the same geographical area (Central Harbin) were recruited within the same period from among patients who were attending vascular 
Li et al.: ADAM17 Polymorphisms and Abdominal Aortic Aneurysm

outpatient clinics and those who were admitted for surgery at the First Affiliated Hospital of Harbin Medical University.

\section{DNA Isolation and Genotyping}

Genomic DNA was isolated from peripheral blood using the EZ-10 Spin Column Whole Blood Genomic DNA Isolation Kit (Sangon Biotech, Shanghai, China), according to manufacturer's instruction.

The two ADAM17 promoter polymorphisms (rs12692386 and rs1524668) were selected according to the results of the ADAM17 promoter sequencing reported by Wang et al. [14]. The polymorphisms of genes were analyzed by SNaPshot Multiplex Kit (Applied Biosystems Co., Ltd., Foster City, CA, USA). The primers used in the SNaPshot were as follows: rs12692386F: GGCCTAGCCCCTCAATCCTCTT, rs12692386R: TTTTTTTGGTAACGCCAC CTGCCTTC; rs1524668F: AAAAACGTTGGGTACATGTTGCTG, rs1524668R: TTTTTTTTT TTTTTTTTTGGTTTCTTTTCTGAACATCCAGTCACCATA. SNaPshot reactions were carried out in a $10 \mu \mathrm{L}$ final volume containing $5 \mu \mathrm{L} \mathrm{SNaPshot} \mathrm{Multiplex} \mathrm{Kit} \mathrm{(ABI),} 1 \mu \mathrm{L}$ primer mix, $2 \mu \mathrm{L}$ water, and $2 \mu \mathrm{L}$ templates consisting of the multiplex PCR products from the different genes. SNaPshot response procedures: (1) initial denaturation at $96^{\circ} \mathrm{C}$ for $1 \mathrm{~min},(2)$ denaturation at $96^{\circ} \mathrm{C}$ for 10 seconds, (3) annealing at $52^{\circ} \mathrm{C}$ for 5 seconds, (4) extension at $60^{\circ} \mathrm{C}$ for 30 seconds, and (5) for a total of 28 cycles. Amplified samples were stored at $4^{\circ} \mathrm{C}$. Extension products were purified by 1-h incubation with $1 \mathrm{U}$ of shrimp alkaline phosphatase (Takara:Otsu, shiga, Japan) at $37^{\circ} \mathrm{C}$ and $75^{\circ} \mathrm{C}$ for $15 \mathrm{~min}$ to inactivate the enzyme. The purified products $(0.5 \mu \mathrm{L})$ were mixed with $9 \mu \mathrm{L}$ of Hi-Di and $0.5 \mu \mathrm{L}$ Liz120 SIZE STANDARD. Samples were incubated at $95^{\circ} \mathrm{C}$ for 5 min and then loaded on an ABI 3130XL DNA sequence detector for capillary electrophoresis. The experimental results were analyzed with GeneMapper 4.0 (Applied Biosystems Co., Ltd.).

\section{RNA extraction}

A total of 50 human AAA samples were obtained from AAA patients who underwent surgical repair at the Department of Vascular Surgery of the First Affiliated Hospital of Harbin Medical University. The control aortic samples were obtained from 50 autopsy specimens from patients who died of unrelated causes at the First Affiliated Hospital of Harbin Medical University. The study was approved by the Hospital Ethics Committee, and written informed consent was obtained from all subjects.

The aortic specimens were immediately segmented in blocks of 3-4 $\mathrm{mm}$, snap-frozen in liquid nitrogen and stored at $-80^{\circ} \mathrm{C}$ for the later RNA and protein extraction. Total RNA was extracted using the RNAprep pure Blood Kit (TianGen Biotech, Beijing, China) according to the manufacturer's instructions.

\section{Real-time PCR}

The isolated RNA was transcribed into cDNA using the cDNA Synthesis Kit RevertAid (Thermo) according to the manufacturer's instructions. Quantitative real-time PCR was performed using the SYBR Green RT-PCR Kit (Takara). Real-time RT-PCR primers for both human GAPDH and ADAM17 coding sequences were designed using Primer Premier 5 software (Applied Biosystems). The primer sequences used were as follows: ADAM17 sense primer, CTG TGG TGC AAA AGC AGA AA; ADAM17 anti-sense primer, TGC CAA ATG CCT CAT ATT CA; GAPDH sense primer, GAA GGG CTC ATG ACC ACA GTC CAT; and GAPDH anti-sense primer, TCA TTG TCG TAC CAG GAA ATG AGC TT. The ADAM17 and GAPDH mRNA levels were measured in triplicate, and the relative $\mathrm{mRNA}$ quantity was determined by the $2^{\mathrm{ADCCt}}$ method and normalized with respect to the housekeeping gene glyceraldehyde-3-phosphate dehydrogenase (GAPDH). The GAPDH expression used for normalization did not vary significantly among the groups. Real-time PCR was performed using a LightCycler ${ }^{4} 80$ sequence detector system (Roche Applied Sciences). The following PCR conditions were used over 40 cycles: $5 \mathrm{~min}$ at $95^{\circ} \mathrm{C}, 5 \mathrm{sec}$ at $95^{\circ} \mathrm{C}, 20 \mathrm{sec}$ at $59^{\circ} \mathrm{C}$, and $10 \mathrm{sec}$ at $72^{\circ} \mathrm{C}$. The relative expression levels for each sample were calculated based on three technical replicates. The amplification products were validated by melting curve analysis.

\section{Enzyme-linked immunosorbent assay (ELISA)}

Blood specimens for the measurement of serum cytokines concentrations were drawn in EDTAcontaining tubes and centrifuged at low speed, and the plasma aliquots were stored at $-20^{\circ} \mathrm{C}$. Levels of TNF- $\alpha$ were measured in duplicate using Quantikine sandwich ELISA kits (R\&D Systems, Minneapolis, MN, USA) according to the manufacturer's instructions. 
Ultrasound Measurement of Abdominal Aortic Aneurysms

AAA size was measured as the greatest diameter of the infrarenal aorta by using ultrasound. The maximum transverse and anteroposterior diameter of the infrarenal abdominal aorta was measured by an experienced vascular sonographer using a color Doppler ultrasound (GE Healthcare Technologies, Ultrasound, Milwaukee, WI, USA) with a $5 \mathrm{MHz}$ transducer. An abdominal aortic aneurysm is defined as an enlargement of the aorta of at least 1.5 times its normal diameter or greater than $3 \mathrm{~cm}$ diameter in total. The reproducibility of aortic measurements is regularly assessed in the Vascular Surgery Department.

\section{Statistical Analyses}

Statistical analyses were performed with SPSS software, version 19.0 (IBM, Armonk, NY, USA). The clinical data are expressed as the means \pm standard deviation (SD) for continuous variables and as the medians and percentage for quantitative variables; a chi-squared test and Student's t-test were used to compare variables between the two groups. Allele frequencies were calculated from the genotypes of all of the subjects. The allele and genotype frequencies of $A D A M 17$ between the patients and control subjects were compared using Fisher's exact test or the Chi-squared test. Deviation of the genotype or allele frequency was assessed using Hardy-Weinberg equilibrium (HWE). The relationships between different genotypes of ADAM17 and AAA were evaluated by an ANOVA. The comparisons of ADAM17 expression levels between the patients and controls were evaluated using Student's t-test for the normally distributed data or a Mann-Whitney-U test for the non-parametric data. Statistical differences with respect to plasma TNF- $\alpha$ level between independent groups were calculated using the Kruskal-Wallis test. The associations of gene polymorphisms with aortic diameter were analyzed with a multiple linear regression model (SAS, version 6.12; SAS Institute Inc., Cary, NC, USA). P values less than 0.05 were considered to be statistically significant.

\section{Results}

\section{Demographic characteristics}

The demographic characteristics of all the participants in the study are summarized in Table 1 . Of the 622 participants, 316 were patients with AAA and 306 were healthy controls. There were no significant differences between the patients and controls with respect to age, diabetes, or hypertension. The mean age was 70.3 years old ( \pm 6.8 years) for the AAA subjects and 69.9 years old ( \pm 7.2 years) for the control subjects. Significant gender and aortic diameter differences in the occurrence of AAA were observed. Consistent with other reports $[17,18]$, AAA was substantially more common among men than among women in this study. Significant differences were also found for risk factors including smoking status and dyslipidemia.

\section{ADAM17 gene polymorphisms and the risk of AAA}

The genotype and allele frequencies of the ADAM17 polymorphisms are shown in Table 2. No deviation from the Hardy-Weinberg equilibrium was observed for the polymorphisms that were examined among the genotype distributions between the AAA and control subjects (data not shown).

A comparison of genotype distributions between the AAA and control subjects using the $\chi 2$-test revealed a statistical association $(\mathrm{P}=0.0096)$ between the ADAM17 rs12692386 polymorphism and the risk of developing AAA. In a dominant model (AA+AG versus GG), no significant difference was detected between the AAA and control groups $(\mathrm{P}=0.11)$. However, in a recessive model (AA versus $A G+G G$ ), a significant difference was observed in the AAA group when compared with the controls $(\mathrm{P}=0.022)$. However, the rs12692386 G allele did not show a significant difference in the AAA group when compared with the controls $(\mathrm{P}=0.14)$. In contrast, there was no significant association between the ADAM17 rs1524668 polymorphism and the risk of developing AAA $(\mathrm{P}=0.77)$. Accordingly, the frequency of the rs1524668 polymorphism was not significantly different between the two groups in either the dominant $(\mathrm{P}=0.58)$ or recessive models $(\mathrm{P}=0.86)$. Additionally, there were no 
Li et al.: ADAM17 Polymorphisms and Abdominal Aortic Aneurysm

Table 1. Characteristics of AAA cases and controls. Continuous data are presented as mean $\pm \mathrm{SD}$, median (range) or $\mathrm{n}(\%)$

\begin{tabular}{lccc}
\hline Variables & AAA $(\mathrm{n}=316)$ & control $(\mathrm{n}=306)$ & P value \\
\hline Mean age (years) & $70.3 \pm 6.8$ & $69.9 \pm 7.2$ & 0.79 \\
Male/female & $242 / 74$ & $155 / 151$ & $<0.001$ \\
Aortic diameter (mm) & $55.1 \pm 8.9$ & $21.8 \pm 5.7$ & $<0.001$ \\
Smokers, n (\%) & $173(54.7)$ & $104(34.0)$ & $<0.001$ \\
Diabetes, n (\%) & $65(20.6)$ & $53(17.32)$ & 0.28 \\
Hypertension, n (\%) & $121(38.3)$ & $105(34.3)$ & 0.71 \\
Dyslipidemia n (\%) & $148(46.8)$ & $106(34.6)$ & $<0.001$ \\
\hline
\end{tabular}

Table 2. Frequencies of ADAM17genotypes and alleles in AAA patients and controls

\begin{tabular}{cllll}
\hline Genotypes & $\begin{array}{l}\text { AAA patients } \\
\mathrm{n}=316(\%)\end{array}$ & $\begin{array}{l}\text { Controls } \\
\mathrm{n}=306(\%)\end{array}$ & OR (95\%CI) & $\begin{array}{l}P \\
\text { value }\end{array}$ \\
\hline rs12692386 & $156(49.3)$ & $179(58.6)$ & & 0.0096 \\
AA & $155(49.1)$ & $116(38.0)$ & & \\
GG & $5(1.6)$ & $11(3.4)$ & & 0.11 \\
Dominant model AA/AG vs GG & $311(98.4)$ & $295(96.4)$ & $2.32(0.80-6.76)$ & 0.022 \\
Recessive model AA vs AG/GG & $160(50.6)$ & $127(41.5)$ & $0.69(0.50-0.95)$ & 0.14 \\
A allele & $467(73.9)$ & $474(77.5)$ & $1.000($ reference $)$ & $1.21(0.94-1.57)$ \\
G allele & $165(26.1)$ & $138(22.5)$ & & 0.77 \\
rs1524668 & & & & \\
AA & & & & \\
AC & $241(76.27)$ & $239(78.98)$ & & 0.58 \\
CC & $73(23.10)$ & $66(20.68)$ & & 0.86 \\
Dominant model AA/AC vs CC & $2(0.63)$ & $1(0.34)$ & & 0.56 \\
Recessive model AA vs AC/ CC & $75(23.73)$ & $67(21.90)$ & $0.98(0.77-1.24)$ & \\
A allele & $555(87.82)$ & $544(88.89)$ & $1.000($ reference $)$ & \\
C allele & $77(12.18)$ & $68(11.11)$ & $1.1(0.78-1.57)$ & \\
\hline
\end{tabular}

statistically significant differences in the frequency of the $\mathrm{C}$ allele of the ADAM17 rs1524668 polymorphism between the AAA group and the control group $(\mathrm{P}=0.56)$.

Associations between ADAM17 gene polymorphisms and demographic characteristics

The associations between ADAM17 gene polymorphisms and demographic characteristics are shown in Tables 3 and 4. In an analysis stratified by gender, increased risk was associated with the variant genotype rs12692386 AG in male patients ( $\mathrm{P}=0.0058)$. No differences in the ADAM17 rs12692386 genotype and allele frequencies were observed between AAA cases and control subjects when the sample was stratified by smoking status. Unexpectedly, when the sample was stratified according to either gender or smoking status, a borderline association in the ADAM17 rs1524668 genotype was determined between AAA patients and the controls $(\mathrm{P}=0.045$ and $\mathrm{P}=0.035$, respectively). However, when the sample was stratified by either age, diabetes or hypertension, no significant differences in the genotype or allele frequencies were detected between the AAA and control groups for either of the two ADAM17 polymorphisms (rs12692386 and rs1524668) $(\mathrm{P}>0.05)$.

Effects of ADAM17 gene polymorphisms on ADAM17 expression

A comparative analysis was performed on the ADAM17 mRNA expression levels among 50 AAA patients' samples and 50 control samples. Consistent with a previous report [9], the mean value of the ADAM17 mRNA expression was significantly higher in the samples of AAA patients than in the controls ( $\mathrm{P}=0.018)$ (Fig. 1). 
Table 3. A comparision between the baseline characteristics of the rs12692386 genotypes and alleles in the AAA patient and control groups. $\mathrm{P}_{\mathrm{A}}$ : $\mathrm{p}$ value of the difference in genotype between the case and control groups. $\mathrm{P}_{\mathrm{G}}$ : $\mathrm{p}$ value of the difference in alleles between the case and control groups

\begin{tabular}{|c|c|c|c|c|c|c|c|c|c|c|c|c|}
\hline \multirow[t]{3}{*}{ Characteristics } & \multicolumn{5}{|c|}{ rs12692386 AAA patient group } & \multicolumn{5}{|c|}{ rs12692386 control group } & \multirow{3}{*}{$P_{G}$} & \multirow{3}{*}{$P_{A}$} \\
\hline & \multicolumn{3}{|c|}{ Genotype n (\%) } & \multicolumn{2}{|c|}{ Allele n (\%) } & \multicolumn{2}{|c|}{ Genotype n (\%) } & \multicolumn{3}{|c|}{ Allele $\mathrm{n}(\%)$} & & \\
\hline & $\mathrm{AA}$ & $A G$ & GG & A & G & $\mathrm{AA}$ & $A G$ & GG & A & G & & \\
\hline \multicolumn{13}{|l|}{ Age } \\
\hline$\geq 70$ years & $80(44.7)$ & $97(54.2)$ & $2(1.1)$ & $257(71.8)$ & $101(28.2)$ & $90(57.0)$ & $62(39.2)$ & $6(3.8)$ & $242(76.6)$ & $74(23.4)$ & 0.13 & 0.20 \\
\hline$<70$ years & $75(54.7)$ & $59(43.1)$ & $3(2.2)$ & $209(76.3)$ & $65(23.7)$ & $88(59.5)$ & $55(40.5)$ & $5(3.4)$ & $231(78.0)$ & $65(22.0)$ & 0.90 & 0.67 \\
\hline $\begin{array}{l}\text { Gender } \\
\text { Male }\end{array}$ & $116(47.9)$ & $125(51.7)$ & $1(0.4)$ & $357(73.8)$ & $127(26.2)$ & $94(60.6)$ & $56(36.1)$ & $5(3.2)$ & $244(78.7)$ & $66(21.3)$ & $\begin{array}{l}0.005 \\
8\end{array}$ & 0.98 \\
\hline Female & $39(52.7)$ & $31(41.9)$ & $4(5.4)$ & $109(73.6)$ & $39(26.4)$ & $84(55.6)$ & $61(40.4)$ & $6(4.0)$ & $229(75.8)$ & $73(24.2)$ & 0.67 & 0.39 \\
\hline \multicolumn{13}{|l|}{ Smoking } \\
\hline Yes & $78(45.1)$ & $91(52.6)$ & $4(2.3)$ & $247(71.4)$ & $99(28.6)$ & $61(58.7)$ & $39(37.5)$ & $4(3.8)$ & $161(77.4)$ & $47(22.6)$ & 0.19 & 0.14 \\
\hline No & $77(53.8)$ & $65(45.5)$ & $1(0.7)$ & $219(76.6)$ & $67(23.4)$ & $117(57.9)$ & $78(38.6)$ & $7(3.5)$ & $312(77.2)$ & $92(22.8)$ & 0.97 & 0.96 \\
\hline \multicolumn{13}{|l|}{ Diabetes } \\
\hline Yes & $32(49.2)$ & $30(46.2)$ & $3(4.6)$ & $94(72.3)$ & $36(27.7)$ & $30(56.6)$ & $21(39.6)$ & $2(3.8)$ & $81(76.4)$ & $25(23.6)$ & 0.085 & 0.68 \\
\hline No & $123(49.0)$ & $126(50.2)$ & $2(0.8)$ & $372(74.1)$ & $130(25.9)$ & $148(58.5)$ & $96(37.9)$ & $9(3.6)$ & $392(77.5)$ & $114(22.5)$ & 0.97 & 0.81 \\
\hline \multicolumn{13}{|l|}{ Hypertension } \\
\hline Yes & $58(47.9)$ & $61(50.4)$ & $2(1.7)$ & $177(73.1)$ & $65(26.9)$ & $61(59.1)$ & $39(37.1)$ & $5(4.8)$ & $\begin{array}{l}161 \\
(76.7)\end{array}$ & $49(23.3)$ & 0.95 & 0.79 \\
\hline No & $97(49.7)$ & $95(48.8)$ & $3(1.5)$ & $289(74.1)$ & $101(25.9)$ & $117(58.2)$ & $78(38.8)$ & $6(3.0)$ & $\begin{array}{l}312 \\
(77.6)\end{array}$ & $90(22.4)$ & 0.72 & 0.79 \\
\hline
\end{tabular}

Table 4. A comparision between the baseline characteristics of the rs1524668 genotypes and alleles in the AAA patient and control groups. $\mathrm{P}_{\mathrm{A}}$ : $\mathrm{p}$ value of the difference in genotype between the case and control groups. $\mathrm{P}_{\mathrm{G}}$ : $\mathrm{p}$ value of the difference in alleles between the case and control groups

\begin{tabular}{|c|c|c|c|c|c|c|c|c|c|c|c|c|}
\hline \multirow[t]{3}{*}{ Characteristics } & \multicolumn{5}{|c|}{ rs1524668 AAA patient group } & \multicolumn{5}{|c|}{ rs1524668 control group } & \multirow{3}{*}{$\mathrm{P}_{\mathrm{G}}$} & \multirow{3}{*}{$\mathrm{P}_{\mathrm{A}}$} \\
\hline & \multicolumn{3}{|c|}{ Genotype n (\%) } & \multicolumn{2}{|c|}{ Allele n (\%) } & \multicolumn{2}{|c|}{ Genotype n (\%) } & & \multicolumn{2}{|c|}{ Allele $\mathrm{n}(\%)$} & & \\
\hline & $\mathrm{AA}$ & $\mathrm{AC}$ & $\mathrm{CC}$ & $\mathrm{A}$ & C & $\mathrm{AA}$ & $\mathrm{AC}$ & $\mathrm{CC}$ & $\mathrm{A}$ & C & & \\
\hline \multicolumn{13}{|l|}{ Age } \\
\hline$\geq 70$ years & $140(78.2)$ & $38(21.2)$ & $1(0.6)$ & $318(88.8)$ & $40(11.2)$ & $119(75.3)$ & $39(24.7)$ & 0 & $277(87.7)$ & $39(12.3)$ & 0.58 & 0.37 \\
\hline$<70$ years & $102(74.5)$ & $33(24.1)$ & $2(1.4)$ & $237(86.5)$ & $37(13.5)$ & $114(77.0)$ & $33(22.3)$ & $1(0.7)$ & $261(88.2)$ & $35(11.8)$ & 0.5 & 0.84 \\
\hline \multicolumn{13}{|l|}{ Gender } \\
\hline Male & $192(79.3)$ & $47(19.4)$ & $3(12.4)$ & $431(89.0)$ & $53(11.0)$ & $119(76.8)$ & $35(22.6)$ & $1(0.6)$ & $273(88.1)$ & $37(11.9)$ & 0.045 & 0.087 \\
\hline Female & $50(67.6)$ & $24(32.4)$ & 0 & $124(83.8)$ & $24(16.2)$ & $114(75.5)$ & $37(24.5)$ & 0 & $265(87.7)$ & $37(12.3)$ & 0.57 & 0.90 \\
\hline \multicolumn{13}{|l|}{ Smoking } \\
\hline Yes & $139(80.3)$ & $31(17.9)$ & $3(1.8)$ & $309(89.3)$ & $37(10.7)$ & $83(79.8)$ & $21(20.2)$ & 0 & $187(89.9)$ & $21(10.1)$ & 0.035 & 0.21 \\
\hline No & $103(72.0)$ & $40(28.0)$ & 0 & $246(86.0)$ & $40(14.0)$ & $150(74.3)$ & $51(25.2)$ & $1(0.5)$ & $351(86.9)$ & 53(13.1) & 0.46 & 0.28 \\
\hline \multicolumn{13}{|l|}{ Diabetes } \\
\hline Yes & $53(81.5)$ & $12(18.5)$ & 0 & $118(90.8)$ & $12(9.2)$ & $41(77.4)$ & $12(22.6)$ & 0 & $94(88.7)$ & $12(11.3)$ & 0.44 & 0.25 \\
\hline No & $189(75.3)$ & $59(23.5)$ & $3(1.2)$ & $437(87.1)$ & 65(12.9) & $192(75.9)$ & $60(23.7)$ & $1(0.4)$ & $444(87.7)$ & $62(12.3)$ & 0.89 & 0.79 \\
\hline \multicolumn{13}{|l|}{ Hypertension } \\
\hline Yes & $95(78.5)$ & $25(20.7)$ & $1(0.8)$ & $215(88.8)$ & $27(11.2)$ & $81(77.1)$ & $24(22.9)$ & 0 & $186(88.6)$ & $24(11.4)$ & 0.81 & 0.53 \\
\hline No & $147(75.4)$ & $46(23.6)$ & $2(1.0)$ & $340(87.2)$ & $50(12.8)$ & $152(75.6)$ & $48(23.9)$ & $1(0.5)$ & $352(87.6)$ & $50(12.4)$ & 0.75 & 0.72 \\
\hline
\end{tabular}

In addition, we assessed whether there was an association between the mean values of ADAM17 mRNA levels and the ADAM17 genotype in AAA patients. The results are presented in Fig. 2. A significant increase in ADAM17 mRNA expression was observed in AAA patients who carried the mutated rs12692386 AG ADAM17 genotype $(\mathrm{P}=0.035)$, while in control subjects, no association was determined between the individuals with the major AA genotype and those who carried the mutated AG genotype $(\mathrm{P}>0.05$ ) (Fig. 2A). A similar trend, although not significant, was observed in patients carrying the rs12692386 GG ADAM17 genotype $(\mathrm{P}=0.081)$ (Fig. 2A). With regard to the rs1524668 polymorphism, no statistically significant 
Fig. 1. Mean values \pm SD of relative ADAM17 mRNA expression for AAA cases $(n=50)$ and controls $(n=50)$. The blank and black boxes represent the relative ADAM17 expression levels in the control and AAA samples, respectively. The median is indicated by a bar across the box. ${ }^{*} \mathrm{P}=0.018$ when comparing relative ADAM17 mRNA expression levels between the AAA and control samples.

\section{$\mathrm{A}$}
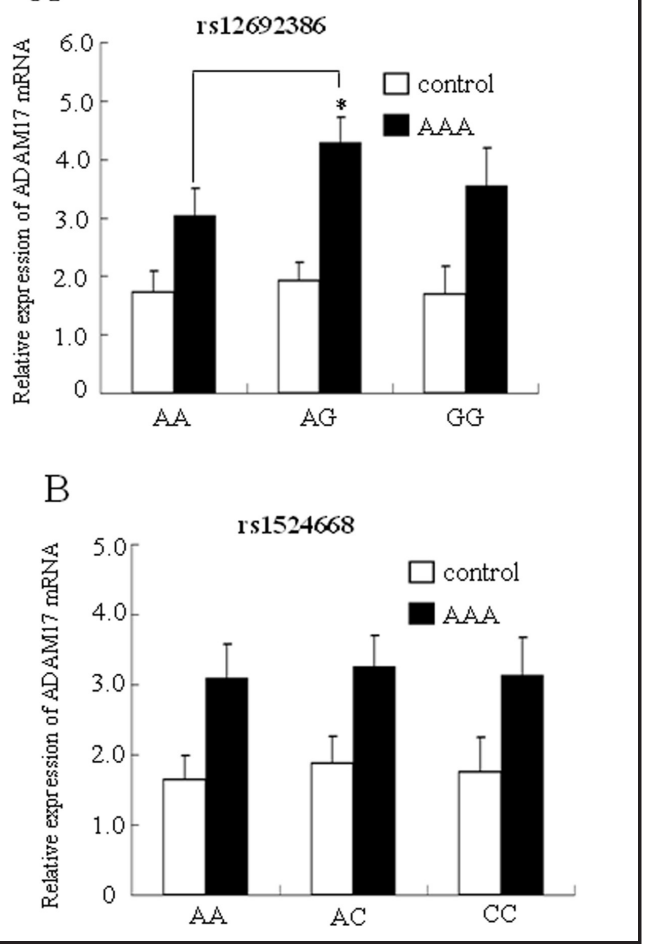

Fig. 2. Mean values \pm SD of ADAM17 mRNA expression from the control samples $(n=50)$ and the AAA tissues $(n=50)$ stratified according to three different genotypes of the ADAM17 rs12692386 and rs1524668 polymorphisms, respectively. Realtime PCR analysis of ADAM17 mRNA expression in tissues derived from the AAA patients and controls with rs12692386 AA, AG and GG genotypes (A) and rs1524668 AA, AC and CC genotypes (B). ${ }^{*} \mathrm{P}=0.035$.
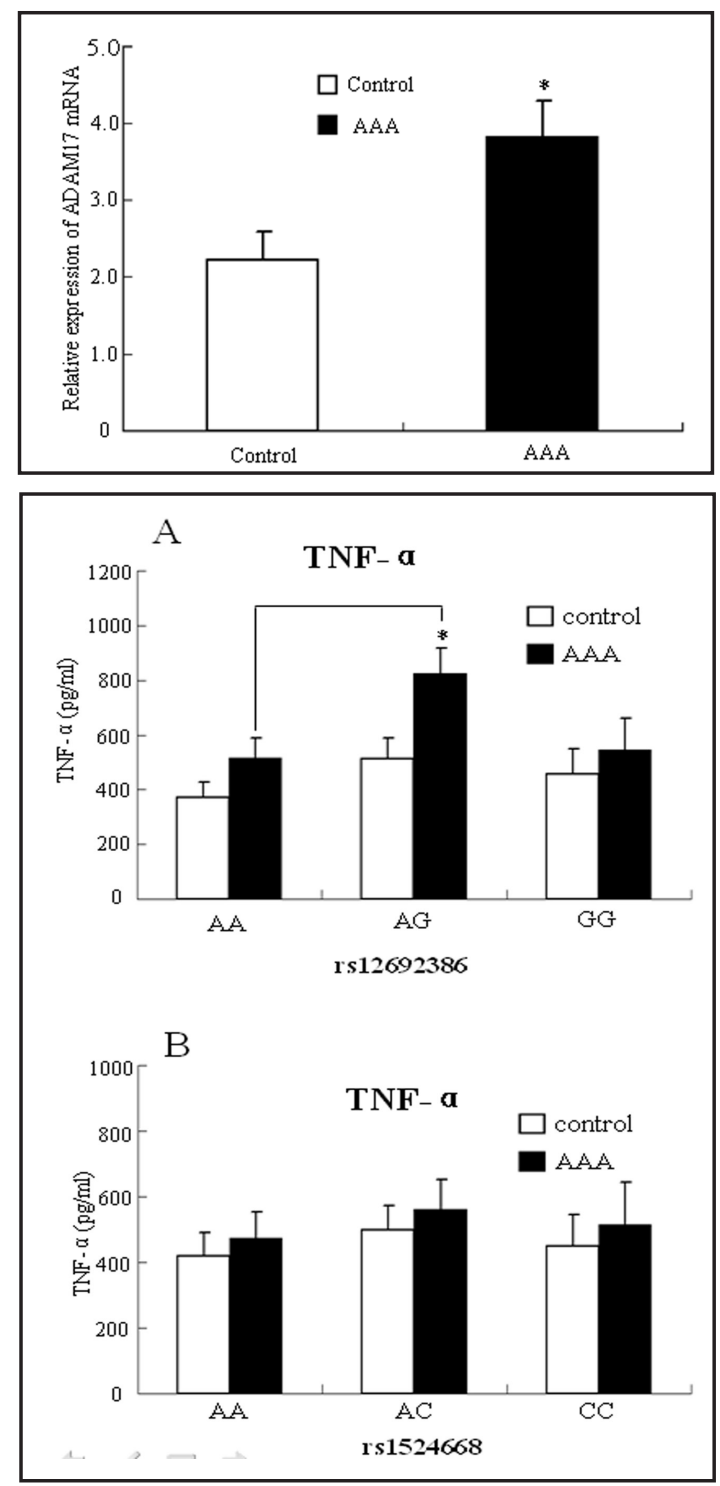

Fig. 3. TNF- $\alpha$ production stratified according to three different genotypes of the ADAM17 rs12692386 and rs1524668 polymorphisms. Serum TNF- $\alpha$ derived from the AAA patients and controls with rs12692386 AA, AG and GG genotype (A) and rs1524668 AA, AC and CC genotypes (B) were measured by ELISA. Data are shown as mean $\pm \mathrm{SD}$. ${ }^{*} \mathrm{P}=0.042(\mathrm{~A})$.

difference in ADAM17 mRNA expression was determined between the population carrying the mutated $\mathrm{AC}$ or $\mathrm{CC}$ genotype and the population carrying the major AA genotype in either the AAA patients or control subjects (Fig. 2B).

Effects of ADAM17 gene polymorphisms on TNF- $\alpha$ expression

Since ADAM17 is a TNF- $\alpha$-converting enzyme, another experiment was performed to determine whether the ADAM17 gene polymorphisms had an effect on TNF- $\alpha$ production in 
Fig. 4. Mean values \pm SD of AAA diameter stratified according to the presence of mutated alleles of the ADAM17 rs12692386 and rs1524668 polymorphisms (A),the rs12692386 polymorphism and gender (B) and the rs1524668 polymorphism and gender (C). $\mathrm{P}>0.05$.

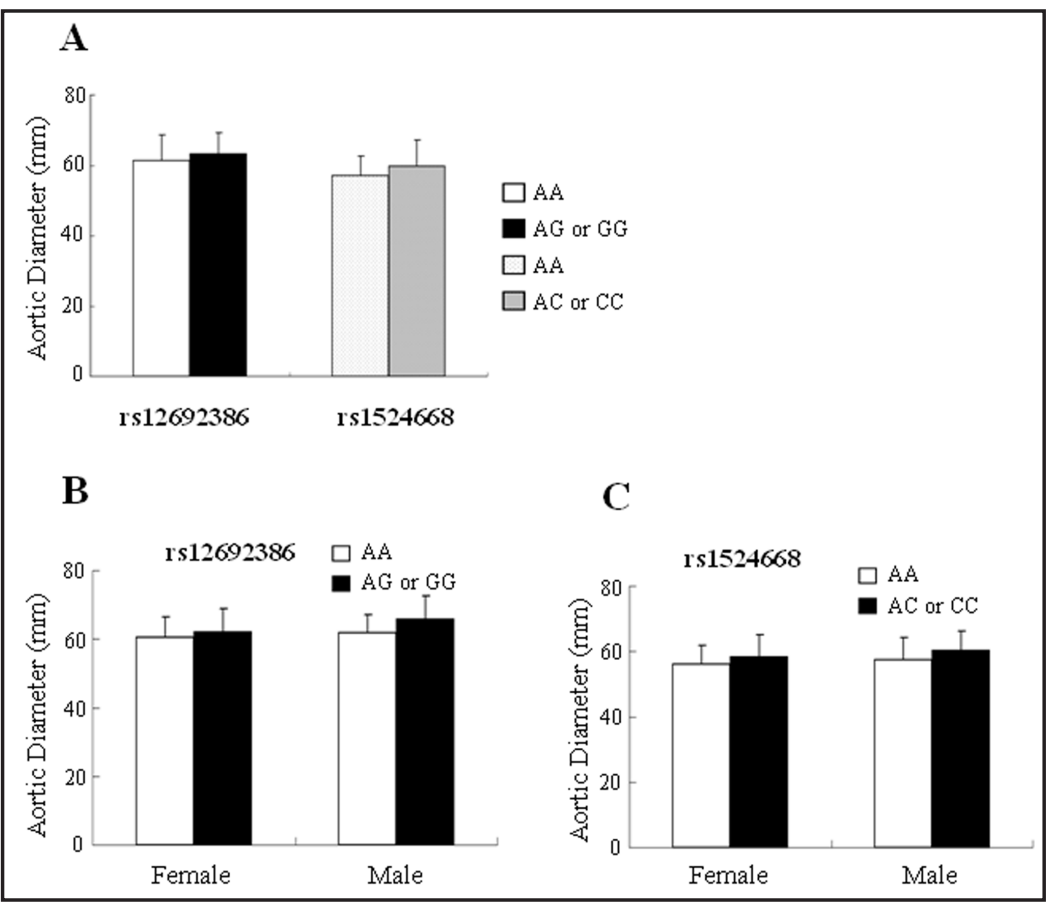

controls and AAA patients. The results are shown in Fig. 3. Generally, the plasma expression levels of TNF- $\alpha$ in AAA patients were significantly higher than in controls $(\mathrm{P}<0.05)$ (Fig. 3). The AAA patients carrying the rs12692386 AG ADAM17 genotype presented with higher plasma TNF- $\alpha$ levels compared to the homologous AA or GG group ( $\mathrm{P}=0.042$ ) (Fig. 3A). The expression of TNF- $\alpha$ did not vary significantly between the population carrying the mutated $\mathrm{AC}$ or $\mathrm{CC}$ genotype and the population carrying the major AA genotype in either the AAA patients or control subjects ( $\mathrm{P}>0.05$ ) (Fig. 3B).

\section{Effects of ADAM17 gene polymorphisms on aortic diameter}

It is well established that the aortic diameter in AAA cases is an important predictor of rupture. In the present study, associations of the ADAM17 gene polymorphisms with AAA aortic diameter were explored, and the results are shown in Fig. 4. The mean value of the AAA aortic diameter in the AAA patients with the variant genotypes rs12692386 (AG and GG) and rs1524668 (AC and CC) was not significantly different from that of patients with the major AA genotype ( $\mathrm{P}>0.05$ ) (Fig. $4 \mathrm{~A})$. We also evaluated the effect of the two ADAM17 polymorphisms on aortic diameter in patients and controls stratified by gender, and found no difference in aortic diameter either in female or male patients $(\mathrm{P}>0.05)$ (Fig. 4B and Fig. 4C).

\section{Discussion}

In this hospital-based case control study, we show for the first time that the rs12692386 polymorphism in the promoter region of $A D A M 17$ is associated with AAA, while the rs1524668 ADAM17 polymorphism is not associated with AAA. Additionally, the rs12692386 $A>G$ polymorphism in the promoter region may affect the expression of ADAM17.

There is increasing evidence of the potential impact of members of the ADAM family on the pathogenic mechanisms that lead to AAA. ADAMs 8, 9, 10,12, 15 and 17 were found to be expressed in both AAA and control aorta [19], and ADAM17 (TACE) was upregulated in human AAA aortic specimens and was associated with chronic inflammation, increased neoangiogenesis and ECM disruption [9]. The role that ADAM17 plays in the inflammation and 
proteolytic degradation of ECM in the vessel wall markedly contributes to the formation and rupture of an AAA [5, 6]. ADAM17 is capable of cleaving several other cell surface molecules, such as TNF- $\alpha$, TNFR1 and TNFR2, as well as IL-6R and IL-1RII, which are considered to be involved in inflammatory processes of the vasculature [10]. The progression of AAA rupture can result from excessive degradation of the extracellular matrix components of the vessel wall, especially that of elastic and collagen fibers. As active metalloproteinases, ADAMs bind to various integrins and can degrade components of the ECM; ADAM17, which acts on collagen XVII as a substrate, may be involved in this process [20]. Additionally, ADAM17 has been reported to promote the transcription of MMP-2, which is thought to play a crucial role in ECM degradation [21]. Thus, increased expression of ADAM17 may facilitate the proliferation and invasive characteristics of inflammatory cells and the disruption of the ECM and therefore contribute to AAA progression [22]. The observations that ADAM17 is present in aortic aneurysm tissue and that its expression is significantly increased in aneurysms have confirmed its involvement in AAA pathogenesis. Despite these advances, the ADAM17 alleles that contribute to AAA remain undiscovered.

To date, only three genome-wide association studies (GWAS) for AAAs have been reported [23-25]. However, the reported odds ratios (ORs) for the identified risk alleles in these studies are not in agreement with the strong heritability patterns that have been observed in epidemiologic studies, which suggests that there are multiple risk loci associated with the disease and many remain unidentified [26]. Previous reports have investigated functional and nonfunctional single nucleotide polymorphisms (SNPs) in patients with aneurysmal disease $[27,28]$. However, no single genetic polymorphism or defect has been identified as a common denominator for AAAs. In our case control study, we show for the first time that the rs12692386 ADAM17 polymorphism is associated with the risk of developing AAA, while the rs1524668 ADAM17 polymorphism is not associated with AAA. Carriers of the rs12692386 AG genotype showed a significantly greater prevalence of AAA than subjects with the AA or GG genotype. Our findings suggest that the rs12692386 polymorphism of ADAM17 may serve as a genetic marker for predicting the occurrence of AAA in high-risk subjects.

AAA has a complex pathophysiology whereby both environmental and genetic factors play important roles [26]. Male sex is an established contributing factor for AAA, and males form AAAs four to five times more often than females [29]. Adriana et al. reported that estrogen receptor alpha was increased in the aortic wall and correlated inversely with MMP activity and AAA formation [30]. Estrogen decreases inflammation, which suggests a protective role for circulating estrogens during AAA formation in females. In addition to estrogens, nitric oxide (NO) and reactive oxygen species are circulating factors that may be responsible for gender differences in AAA formation [31]. Being male is not only genetic but also affects environmental risk factors, such as smoking. Accumulating epidemiological evidence repeatedly confirmed that smoking is a significant risk factor for AAA [26]. The relative risk for current smokers is at least 2-fold, and the association of having ever smoked with AAA is 2.5 times greater than the association of having ever smoked with CHD [26, 32]. Continued smoking also results in increased AAA expansion, a greater risk of rupture and a worse prognosis [33]. Smokers have elevated levels of elastase activity in the arterial wall, which leads to weakening of the aorta [34]. Stolle et al. reported that cigarette mainstream smoke could enhance the proteolytic activity of MMPs, including MMP-2 and -9, induced by Ang II and accelerate both the formation and severity of AAAs in apoE-/- mice [35]. Additionally, nicotine, a principal tobacco alkaloid, was shown to play a proinflammatory role in vasculature in vivo and in vitro [36]. Together, these findings suggest that cigarette smoke may stimulate the formation and progression of AAAs. In our present study, when the rs12692386 genotype and allele frequency of ADAM17 was further stratified by age, gender, smoking status, diabetes and hypertension, an increased risk was found in the male subgroup of AAA patients when compared to control subjects. A significant association in the ADAM17 rs1524668 genotype was also determined between AAA patients and the controls in males and in the smoking subgroup. Thus, it is highly likely that environmental risk factors, gender and smoking interact with an ADAM17 genetic predisposition for the development of AAA. 
In the present study, we employed real-time PCR to detect ADAM17 mRNA expression in human AAA tissues. Consistent with a previous study [9], the expression of ADAM17 in human AAA samples was significantly increased in the AAA patients in comparison to the control subjects. We also found that the individuals who carried the ADAM17 rs12692386 mutated AG genotype had higher ADAM17 mRNA expression levels compared with the population that carried the homologous AA or GG genotype. The rs12692386 A>G polymorphism in the ADAM17 promoter region could be a functional SNP. The mechanism by which the rs12692386 ADAM17 polymorphism contributes to elevated ADAM17 expression is unknown. Given that the two SNPs map to promoter regions, transcriptional enhancers and other regulatory elements in the promoter regions might play a regulatory role. Moreover, computational analysis of the rs12692386 A>G polymorphism by MAPPER indicated a potential allele-specific binding site for the upstream stimulatory factor (USF) transcription factor [37]. The rs12692386 A $>$ G ADAM17 polymorphism could affect binding to USF and thereby regulate gene expression.

The formation and expansion of an AAA result from extracellular matrix degradation, increased proteolytic activity, and widespread inflammation. Logically, treatment with anti-inflammatory drugs could inhibit the expansion of AAAs and reduce AAA-size [7]. A platelet P2Y12 receptor inhibitor reduces vascular inflammation and thus suppresses AAA expansion in apoE-/- mice [38]. Knockout of the ADAM17 inhibitor, Tissue Inhibitor of Metalloproteinase 3 (TIMP3) in vivo exacerbates AAA expansion and inflammation in the abdominal aorta [39]. These lines of evidence indicate that inhibiting ADAM17 or inflammation may alleviate AAA expansion. Our present study did not demonstrate any significant association between aortic diameter and the ADAM17 polymorphisms, even taking the gender status into consideration, which suggests that these polymorphisms do not affect AAA growth. A previous study concerning an MMP-2 $1306 \mathrm{C} / \mathrm{T}$ polymorphism also found no association between the polymorphisms and aortic diameter [40]. Our findings provide little support for ADAM17 polymorphisms contributing to AAA expansion. Nevertheless, the exact mechanism of AAA expansion remains under investigation. AAA expansion may involve multiple factors, and the positive effect associated with ADAM17 polymorphisms may be counteracted by other factors (hormonal factors, smoking, hypertension, diabetes and other risk factors).

Despite the results described above, limitations of the present study should to be acknowledged. The small number of participants in this study was insufficient and therefore may have led to nonrepresentative results. Thus, this limitation may have suppressed a true relationship due to a type II statistical error. However, notably, the prevalence of AAAs in the Chinese population is comparatively lower than that in Caucasian populations [41]. The mechanism that underlies this interesting ethnic difference remains unclear. Selection bias in the patient or control populations may not be entirely excluded. Specifically, controls were not matched for environmental risk factors of aortic aneurysms because we compared the allele frequencies in the samples to those in the population from which they came. Selection of controls based upon specific phenotypes (such as smoker/non-smoker) would in itself risk introducing bias and would therefore be irrelevant. The other risk factors in the study group, such as age, gender, smoking, hypertension, diabetes or hyperlipaemia may have complicated the association between ADAM17 polymorphisms and AAA. In addition, other functional polymorphisms may influence the expression of ADAM17, and their combined effects must be studied to better predict the occurrence, severity, and outcome of AAA. The results obtained in this study require confirmation in independent, larger populations and in different ethnic backgrounds before the conclusions can be considered definitive and useful for estimating an individual's risk for developing AAA.

In conclusion, our study shows for the first time a significant association between the ADAM17 rs12692386 polymorphism and an increased risk of AAA. ADAM17 is therefore a promising new genetic candidate that plays a role in regulating the development of AAA. However, more work is required to further understand the role of ADAM17 in the pathogenesis of AAA and to clarify its prognostic and therapeutic potential. 


\section{Acknowledgements}

This work was supported by funding from the National Nature Science Foundation of China (grant numbers 31171219, 81271213, 81070878, 81271214, 81300929 and 81261120404); the Natural Science Foundation of Guangdong Province, China (No. S2012010008222); and the Science and Technology Innovation Fund of Guangdong Medical College (No. STIF 201101).

\section{Disclosure Statement}

The authors have no actual or potential conflicts of interest related to this manuscript. Appropriate approval was obtained, and appropriate procedures were followed concerning human subjects.

\section{References}

1 Zhang F, Kent KC, Yamanouchi D, Zhang Y, Kato K, Tsai S, Nowygrod R, Schmidt AM, Liu B: Anti-receptor for advanced glycation end products therapies as novel treatment for abdominal aortic aneurysm. Ann Surg 2009;250:416-423.

2 Choke E, Cockerill G, Wilson WR, Sayed S, Dawson J, Loftus I, Thompson MM: A review of biological factors implicated in abdominal aortic aneurysm rupture. Eur J Vasc Endovasc Surg 2005;30:227-244.

-3 Thompson MM, Jones L, Nasim A, Sayers RD, Bell PR: Angiogenesis in abdominal aortic aneurysms. Eur J Vasc Endovasc Surg 1996;11:464-469.

4 Zhang J, Schmidt J, Ryschich E, Mueller-Schilling M, Schumacher H, Allenberg JR: Inducible nitric oxide synthase is present in human abdominal aortic aneurysm and promotes oxidative vascular injury. J Vasc Surg 2003;38:360-367.

5 Kadoglou NP, Liapis CD: Matrix metalloproteinases: Contribution to pathogenesis, diagnosis, surveillance and treatment of abdominal aortic aneurysms. Curr Med Res Opin 2004;20:419-432.

6 Hinterseher I, Erdman R, Elmore JR, Stahl E, Pahl MC, Derr K, Golden A, Lillvis JH, Cindric MC, Jackson K, Bowen WD, Schworer CM, Chernousov MA, Franklin DP, Gray JL, Garvin RP, Gatalica Z, Carey DJ, Tromp G, Kuivaniemi H: Novel pathways in the pathobiology of human abdominal aortic aneurysms. Pathobiology 2013;80:1-10.

7 Lindholt JS, Shi GP: Chronic inflammation, immune response, and infection in abdominal aortic aneurysms. Eur J Vasc Endovasc Surg 2006;31:453-463.

8 Xiong W, MacTaggart J, Knispel R, Worth J, Persidsky Y, Baxter BT: Blocking tnf-alpha attenuates aneurysm formation in a murine model. J Immunol 2009;183:2741-2746.

-9 Kaneko H, Anzai T, Horiuchi K, Kohno T, Nagai T, Anzai A, Takahashi T, Sasaki A, Shimoda M, Maekawa Y, Shimizu H, Yoshikawa T, Okada Y, Yozu R, Fukuda K: Tumor necrosis factor-alpha converting enzyme is a key mediator of abdominal aortic aneurysm development. Atherosclerosis 2011;218:470-478.

$\checkmark 10$ Dreymueller D, Pruessmeyer J, Groth E, Ludwig A: The role of adam-mediated shedding in vascular biology. Eur J Cell Biol 2012;91:472-485.

11 Canault M, Peiretti F, Kopp F, Bonardo B, Bonzi MF, Coudeyre JC, Alessi MC, Juhan-Vague I, Nalbone G: The tnf alpha converting enzyme (tace/adam17) is expressed in the atherosclerotic lesions of apolipoprotein e-deficient mice: Possible contribution to elevated plasma levels of soluble tnf alpha receptors. Atherosclerosis 2006;187:82-91.

12 Canault M, Peiretti F, Mueller C, Kopp F, Morange P, Rihs S, Portugal H, Juhan-Vague I, Nalbone G: Exclusive expression of transmembrane tnf-alpha in mice reduces the inflammatory response in early lipid lesions of aortic sinus. Atherosclerosis 2004;172:211-218.

13 Lu H, Rateri DL, Bruemmer D, Cassis LA, Daugherty A: Novel mechanisms of abdominal aortic aneurysms. Curr Atheroscler Rep 2012;14:402-412. 
Li et al.: ADAM17 Polymorphisms and Abdominal Aortic Aneurysm

14 Wang M, Li Y, Lu Y, Zuo X, Wang F, Zhang Z, Jia J: The relationship between adam17 promoter polymorphisms and sporadic alzheimer's disease in a northern chinese han population. J Clin Neurosci 2010;17:1276-1279.

15 Junyent M, Parnell LD, Lai CQ, Arnett DK, Tsai MY, Kabagambe EK, Straka RJ, Province M, An P, Smith CE, Lee YC, Borecki I, Ordovas JM: Adam17_i33708a>g polymorphism interacts with dietary n-6 polyunsaturated fatty acids to modulate obesity risk in the genetics of lipid lowering drugs and diet network study. Nutr Metab Cardiovasc Dis 2010;20:698-705.

16 Morange PE, Tregouet DA, Godefroy T, Saut N, Bickel C, Rupprecht HJ, Lackner K, Barbaux S, Poirier O, Peiretti F, Nalbone G, Juhan-Vague I, Blankenberg S, Tiret L: Polymorphisms of the tumor necrosis factoralpha (tnf) and the tnf-alpha converting enzyme (tace/adam17) genes in relation to cardiovascular mortality: The atherogene study. J Mol Med (Berl) 2008;86:1153-1161.

17 Saracini C, Bolli P, Sticchi E, Pratesi G, Pulli R, Sofi F, Pratesi C, Gensini GF, Abbate R, Giusti B: Polymorphisms of genes involved in extracellular matrix remodeling and abdominal aortic aneurysm. J Vasc Surg 2012;55:171-179 e172.

18 Armani C, Landi S, Nuti M, Di Stefano R, Balbarini A: Has-1 genetic polymorphism in sporadic abdominal aortic aneurysm. Heart Int 2009;4:e1.

19 Lipp C, Lohoefer F, Reeps C, Rudelius M, Baummann M, Heemann U, Eckstein HH, Pelisek J: Expression of a disintegrin and metalloprotease in human abdominal aortic aneurysms. J Vasc Res 2012;49:198-206.

20 Edwards DR, Handsley MM, Pennington CJ: The adam metalloproteinases. Mol Aspects Med 2008;29:258289.

21 Longo GM, Xiong W, Greiner TC, Zhao Y, Fiotti N, Baxter BT: Matrix metalloproteinases 2 and 9 work in concert to produce aortic aneurysms. Journal of Clinical Investigation 2002;110:625-632.

22 Saftig P, Reiss K: The "a disintegrin and metalloproteases" adam10 and adam17: Novel drug targets with therapeutic potential? Eur J Cell Biol 2011;90:527-535.

-23 Elmore JR, Obmann MA, Kuivaniemi H, Tromp G, Gerhard GS, Franklin DP, Boddy AM, Carey DJ: Identification of a genetic variant associated with abdominal aortic aneurysms on chromosome 3p12.3 by genome wide association. J Vasc Surg 2009;49:1525-1531.

24 Gretarsdottir S, Baas AF, Thorleifsson G, Holm H, den Heijer M, de Vries J-PP, Kranendonk SE, Zeebregts CJ, van Sterkenburg SM, Geelkerken RH: Genome-wide association study identifies a sequence variant within the dab2ip gene conferring susceptibility to abdominal aortic aneurysm. Nature genetics 2010;42:692-697.

25 Bown MJ, Jones GT, Harrison SC, Wright BJ, Bumpstead S, Baas AF, Gretarsdottir S, Badger SA, Bradley DT, Burnand K, Child AH, Clough RE, Cockerill G, Hafez H, Scott DJ, Futers S, Johnson A, Sohrabi S, Smith A, Thompson MM, van Bockxmeer FM, Waltham M, Matthiasson SE, Thorleifsson G, Thorsteinsdottir U, Blankensteijn JD, Teijink JA, Wijmenga C, de Graaf J, Kiemeney LA, Assimes TL, McPherson R, Folkersen L, Franco-Cereceda A, Palmen J, Smith AJ, Sylvius N, Wild JB, Refstrup M, Edkins S, Gwilliam R, Hunt SE, Potter S, Lindholt JS, Frikke-Schmidt R, Tybjaerg-Hansen A, Hughes AE, Golledge J, Norman PE, van Rij A, Powell JT, Eriksson P, Stefansson K, Thompson JR, Humphries SE, Sayers RD, Deloukas P, Samani NJ: Abdominal aortic aneurysm is associated with a variant in low-density lipoprotein receptor-related protein 1 . Am J Hum Genet 2011;89:619-627.

-26 Bjorck M, Wanhainen A: Pathophysiology of aaa: Heredity vs environment. Prog Cardiovasc Dis 2013;56:26.

27 Saratzis A, Abbas AA, Kiskinis D, Melas N, Saratzis N, Kitas GD: Abdominal aortic aneurysm: A review of the genetic basis. Angiology 2011;62:18-32.

28 Thompson AR, Drenos F, Hafez H, Humphries SE: Candidate gene association studies in abdominal aortic aneurysm disease: A review and meta-analysis. Eur J Vasc Endovasc Surg 2008;35:19-30.

29 Katz DJ, Stanley JC, Zelenock GB: Gender differences in abdominal aortic aneurysm prevalence, treatment, and outcome. J Vasc Surg 1997;25:561-568.

-30 Laser A, Ghosh A, Roelofs K, Sadiq O, McEvoy B, Dimusto P, Eliason J, Upchurch GR, Jr.: Increased estrogen receptor alpha in experimental aortic aneurysms in females compared with males. J Surg Res 2014;186:467-474.

31 Hayashi T, Fukuto JM, Ignarro LJ, Chaudhuri G: Basal release of nitric oxide from aortic rings is greater in female rabbits than in male rabbits: Implications for atherosclerosis. Proc Natl Acad Sci U S A 1992;89:11259-11263. 
-32 Lederle FA, Nelson DB, Joseph AM: Smokers' relative risk for aortic aneurysm compared with other smoking-related diseases: A systematic review. J Vasc Surg 2003;38:329-334.

33 Brady AR, Thompson SG, Fowkes FG, Greenhalgh RM, Powell JT: Abdominal aortic aneurysm expansion: Risk factors and time intervals for surveillance. Circulation 2004;110:16-21.

-34 Jaldin RG, Castardelli E, Perobelli JE, Yoshida WB, de Castro Rodrigues A, Sequeira JL, Paiva SA: Morphologic and biomechanical changes of thoracic and abdominal aorta in a rat model of cigarette smoke exposure. Ann Vasc Surg 2013;27:791-800.

-35 Stolle K, Berges A, Lietz M, Lebrun S, Wallerath T: Cigarette smoke enhances abdominal aortic aneurysm formation in angiotensin ii-treated apolipoprotein e-deficient mice. Toxicol Lett 2010;199:403-409.

36 Li ZZ, Dai QY: Pathogenesis of abdominal aortic aneurysms: Role of nicotine and nicotinic acetylcholine receptors. Mediators Inflamm 2012;2012:103120.

37 Dollery CM, Libby P: Atherosclerosis and proteinase activation. Cardiovasc Res 2006;69:625-635.

-38 Liu O, Jia L, Liu X, Wang Y, Wang X, Qin Y, Du J, Zhang H: Clopidogrel, a platelet p2y12 receptor inhibitor, reduces vascular inflammation and angiotensin ii induced-abdominal aortic aneurysm progression. PLoS One 2012; 7:e51707.

39 Basu R, Fan D, Kandalam V, Lee J, Das SK, Wang X, Baldwin TA, Oudit GY, Kassiri Z: Loss of timp3 gene leads to abdominal aortic aneurysm formation in response to angiotensin ii. J Biol Chem 2012;287:44083-44096.

40 Smallwood L, Warrington N, Allcock R, van Bockxmeer F, Palmer LJ, Iacopetta B, Golledge J, Norman PE: Matrix metalloproteinase-2 gene variants and abdominal aortic aneurysm. Eur J Vasc Endovasc Surg 2009;38:169-171.

41 Wang J-A, Chen X-F, Yu W-F, Chen H, Liu X-M, Lin X-F, Tang L-J, Jiang J-J, Dong L, Jiang J: Prevalence of abdominal aortic aneurysms in chinese coronary artery disease patients. EJVES Extra 2008;15:37-38. 